\title{
Response to Letter to the Editor on "Anti-Psoriatic Drug Monomethylfumarate Increases Nuclear Factor Erythroid 2-Related Factor 2 Levels and Induces Aquaporin-3 mRNA and Protein Expression"
}

\author{
Wendy B. Bollag, Inas Helwa, Vivek Choudhary, Xunsheng Chen, and Ismail Kaddour- \\ Djebbar \\ Charlie Norwood VA Medical Center, Augusta, Georgia (V.C., X.C., I.K.-D., W.B.B.); Department of Oral Biology, Augusta \\ University, Augusta, Georgia (I.H., W.B.B.); and Departments of Physiology (V.C., X.C., I.K.-D., W.B.B.) and Medicine \\ (Dermatology) (W.B.B.), Medical College of Georgia, Augusta University, Augusta, Georgia
}

Received November 20, 2017; accepted December 21, 2017

We thank Lai and colleagues for their interest in our work and appreciate the opportunity to respond to their concerns. We agree with them that the mechanism by which monomethylfumarate acts is as yet unclear, which led us to investigate its effects on nuclear factor erythroid 2-related factor 2 (Nrf2) levels. Indeed, we showed that monomethylfumarate increased Nrf2 protein levels, nuclear translocation, and target gene expression (Helwa et al., 2017); these results are consistent with data reported in other systems (Ananth et al., 2013; Promsote et al., 2014; Ahuja et al., 2016). However, we disagree that oxidative stress simply promotes psoriasis lesions since there seems to be insufficient data to determine whether oxidative stress is an etiologic factor for this disease. Clearly, oxidative stress plays a key role since markers of oxidative stress are elevated in psoriatic patients (Kaur et al., 2013; Peluso et al., 2016; Borska et al., 2017), and reduction of oxidative stress with antioxidant supplementation improves the disease (Kharaeva et al., 2009). Antioxidant molecules can also improve psoriasiform lesions in mouse models of psoriasis (see, e.g., An et al., 2016; Zhang et al., 2016; Chen et al., 2017). While we also agree that $\mathrm{T}$ cells are key mediators of psoriasis, there is evidence that other cells may also be involved. Thus, epidermal-specific c-Jun and JunB conditional knockout mice exhibit a psoriasiform skin phenotype that is reduced but not abolished in mice also lacking Rag-2 (Zenz et al., 2005), and therefore $\mathrm{T}$ and B cells, suggesting the importance of keratinocytes. Similarly, treatment of mice with clodronate liposomes to deplete

The contents of this article do not represent the views of the Department of Veterans Affairs or the United States Government.

This Letter to the Editor is in response to "Anti-psoriatic drug monomethylfumarate increases nuclear factor erythroid 2-related factor 2 levels and induces aquaporin-3 mRNA and protein expression" by Helwa I, Choudhary V, Chen X, Kaddour-Djebbar I, and Bollag WB, found in J Pharmacol Exp Ther 2017, 362:243-253.

https://doi.org/10.1124/jpet.117.246595. monocytes/macrophages improves psoriasiform lesions in two genetic mouse models of psoriasis (Stratis et al., 2006; Ward et al., 2011), implicating involvement of these immune cells in the disease process as well. Nevertheless, we agree with Lai and colleagues that additional studies with monomethylfumarate should be performed in $\mathrm{T}$ cells and other immune system components.

As for the role of Nrf2 in keratinocytes, there is evidence that Nrf2 is involved in keratinocyte proliferation, and Lai and colleagues provide several examples of references reporting such results. However, there are also data in the literature to support a contribution of Nrf2 to keratinocyte differentiation. Thus, Nrf2 levels and activation as well as its targets are increased in a differentiation-dependent manner, and Nrf2 overexpression results in upregulation of the keratinocyte differentiation markers K10 and loricrin (Lee et al., 2014). In addition, the genes encoding LCE1 family members and small proline-rich proteins SPRR2D and SPRR2H are direct targets of Nrf2 (Ishitsuka et al., 2016); these proteins are late keratinocyte differentiation markers that contribute to the cornified envelope and thus the barrier function of the epidermis. Finally, in a transgenic mouse model epidermalspecific expression of a dominant-negative Nrf2 mutant that inhibits the activity of Nrf2 and its family members enhances papilloma formation in a two-stage tumorigenesis model (auf dem Keller et al., 2006), suggesting the ability of this transcription factor family to suppress keratinocyte growth. Thus, together these results are consistent with a differentiative effect of Nrf2 and its targets. As is often the case in biology, the answer as to the exact role of Nrf2 in keratinocytes and skin is likely complex and may be dependent on the levels of Nrf2 relative to other members of the Nrf family and/or additional components of the pathway, the extent of exposure to oxidative stress, and possibly other unknown factors. Alternatively, a fine-tuning of the pathway may be necessary 
such that both too much and too little Nrf2 may contribute to epidermal pathologies. Clearly, further study is needed.

Finally, contrary to the statement by Lai and colleagues in their Letter to the Editor, $\mathrm{K} 10$ is not considered to be a "hyperproliferation-associated keratin" but rather a keratin linked to normal differentiation (Moll et al., 2008). [Indeed, note that the articles cited by Lai and colleagues include these phrases: "normal keratins K14 and K10" and "differentiation marker K10" from the abstract of Elango et al. (2015) and "Keratin 6 (K6) and keratin 10 (K10) are markers for epidermal hyperproliferation and differentiation, respectively" from the abstract of Mommers et al. (2000), whereas $\mathrm{K} 10$ is not mentioned in the article by Ramot et al. (2013) since only keratin 6 and 16 are discussed]. Therefore, we argue that the ability of Nrf2 to induce aquaporin-3 (AQP3), which increases K10 promoter activity (Bollag et al., 2007), as well as mRNA and protein levels when AQP3 is re-expressed in AQP3 knockout keratinocytes (Choudhary et al., 2015), also argues for an effect of Nrf2 in differentiation. Nevertheless, we acknowledge that a controversy exists as to the exact role of AQP3 in the epidermis, since Verkman and colleagues have suggested a pro-proliferative role for AQP3 in keratinocytes and skin cancer (Hara-Chikuma and Verkman, 2008a,b; Verkman, 2008). In contrast, we and other investigators have reported data supporting a prodifferentiative role of AQP3 in keratinocytes. Thus, for instance, we observed an increase in the promoter activities of two differentiation markers (K10 and involucrin) upon cotransfection of keratinocytes with AQP3 but not empty vector (Bollag et al., 2007). In addition, siRNA-mediated knockdown experiments in human keratinocytes demonstrate that cells with decreased AQP3 levels exhibit a reduction in their $\mathrm{K} 10$ upregulation in response to an inducer of differentiation (Kim and Lee, 2010). Further support for involvement of AQP3 in differentiation is provided by the facts that differentiating-stimulating PPAR $\gamma$ agonists upregulate AQP3 expression and levels (Jiang et al., 2011) and that AQP3 is induced at early time points of the differentiation mediated by high cell density in human keratinocytes, with a similar time course to that observed for the increase in keratin 1 expression (Guo et al., 2013).

We believe that the resolution to this controversy concerning the function of AQP3 in keratinocytes may be related to whether this glycerol channel is associated with phospholipase D2 (PLD2) to produce phosphatidylglycerol (or is not associated with PLD2 to instead increase glycerol levels for energy production) (Hara-Chikuma and Verkman, 2008b,c). Thus, we previously demonstrated that PLD2 can use the glycerol transported by AQP3 in a transphosphatidylation reaction to form phosphatidylglycerol (Zheng et al., 2003). PLD2 colocalizes with AQP3 in caveolin-rich membrane microdomains in keratinocytes and these two proteins can be coimmunoprecipitated from lysates of these cells (Zheng and Bollinger Bollag, 2003); therefore, we proposed that AQP3 interacts with PLD2 to funnel glycerol to PLD2 for the production of phosphatidylglycerol (Qin et al., 2011). Subsequent studies in which we manipulated this signaling module indicate that this unit can inhibit proliferation and promote early differentiation of epidermal keratinocytes (Bollag et al., 2007). Indeed, direct addition of phosphatidylglycerol, but not a related phospholipid, can inhibit proliferation and/or trigger differentiation of keratinocytes (Bollag et al., 2007; Xie et al., 2014). This idea is also consistent with our findings that AQP3 re-expression in AQP3 knockout keratinocytes increases the protein and mRNA expression of several keratinocyte differentiation markers in a PLD2 activity-dependent fashion (Choudhary et al., 2015). Nevertheless, we continue to examine the role of AQP3 in keratinocytes, the skin, and skin diseases because of the ongoing debate.

In summary, then, it appears that much of the data concerning the involvement of Nrf2 in keratinocyte proliferation and differentiation is conflicting, with results supporting its participation in both processes. In other words, the role of Nrf2 in keratinocytes and the epidermis is complicated, requiring further investigation by interested dermatologic researchers dedicated to deciphering this important signaling system in the skin.

\section{References}

Ahuja M, Ammal Kaidery N, Yang L, Calingasan N, Smirnova N, Gaisin A, Gaisina IN, Gazaryan I, Hushpulian DM, Kaddour-Djebbar I, et al. (2016) Distinct Nrf2 signaling mechanisms of fumaric acid esters and their role in neuroprotection against 1-methyl-4-phenyl-1,2,3,6-tetrahydropyridine-induced experimental Parkinson's-like disease. $J$ Neurosci 36:6332-6351.

An J, Li Z, Dong Y, Ren J, and Huo J (2016) Amentoflavone protects against psoriasis-like skin lesion through suppression of NF- $\kappa$ B-mediated inflammation and keratinocyte proliferation. Mol Cell Biochem 413:87-95.

Ananth S, Babu E, Veeranan-Karmegam R, Bozard Baldowski BR, Boettger T, and Martin PM (2013) Induction of the cystine/glutamate exchanger SLC7A11 in retinal pigment epithelial cells by the antipsoriatic drug monomethylfumarate. Invest Ophthalmol Vis Sci 54:1592-1602.

auf dem Keller U, Huber M, Beyer TA, Kümin A, Siemes C, Braun S, Bugnon P, Mitropoulos V, Johnson DA, Johnson JA, et al. (2006) Nrf transcription factors in keratinocytes are essential for skin tumor prevention but not for wound healing. Mol Cell Biol 26:3773-3784.

Bollag WB, Xie D, Zhong X, and Zheng X (2007) A potential role for the phospholipase D2-aquaporin-3 signaling module in early keratinocyte differentiation: production of a novel phosphatidylglycerol signaling lipid. J Invest Dermatol 127:2823-2831.

Borska L, Kremlacek J, Andrys C, Krejsek J, Hamakova K, Borsky P, Palicka V, Rehacek V, Malkova A, and Fiala Z (2017) Systemic inflammation, oxidative damage to nucleic acids, and metabolic syndrome in the pathogenesis of psoriasis. Int $J$ Mol Sci 18:2238.

Chen H, Lu C, Liu H, Wang M, Zhao H, Yan Y, and Han L (2017) Quercetin ameliorates imiquimod-induced psoriasis-like skin inflammation in mice via the NF- $\kappa \mathrm{B}$ pathway. Int Immunopharmacol 48:110-117.

Choudhary V, Olala LO, Qin H, Helwa I, Pan ZQ, Tsai YY, Frohman MA, KaddourDjebbar I, and Bollag WB (2015) Aquaporin-3 re-expression induces differentiation in a phospholipase D2-dependent manner in aquaporin-3-knockout mouse keratinocytes. J Invest Dermatol 135:499-507.

Elango T, Thirupathi A, Subramanian S, Dayalan H, and Gnanaraj P (2015) Methotrexate normalized keratinocyte activation cycle by overturning abnormal keratins as well as deregulated inflammatory mediators in psoriatic patients. Clin Chim Acta 451:329-337.

Guo L, Chen H, Li Y, Zhou Q, and Sui Y (2013) An aquaporin 3-notch1 axis in keratinocyte differentiation and inflammation. PLoS One 8:e80179.

Hara-Chikuma M and Verkman AS (2008a) Aquaporin-3 facilitates epidermal cell migration and proliferation during wound healing. J Mol Med (Berl) 86:221-231.

Hara-Chikuma M and Verkman AS (2008b) Prevention of skin tumorigenesis and impairment of epidermal cell proliferation by targeted aquaporin-3 gene disruption. Mol Cell Biol 28:326-332.

Hara-Chikuma M and Verkman AS (2008c) Roles of aquaporin-3 in the epidermis. $J$ Invest Dermatol 128:2145-2151.

Helwa I, Choudhary V, Chen X, Kaddour-Djebbar I, and Bollag WB (2017) Antipsoriatic drug monomethylfumarate increases nuclear factor erythroid 2-related factor 2 levels and induces aquaporin-3 mRNA and protein expression. $J$ Pharmacol Exp Ther 362:243-253.

Ishitsuka Y, Huebner AJ, Rice RH, Koch PJ, Speransky VV, Steven AC, and Roop DR (2016) Lce1 family members are Nrf2-target genes that are induced to compensate for the loss of loricrin. J Invest Dermatol 136:1656-1663.

Jiang YJ, Kim P, Lu YF, and Feingold KR (2011) PPARgamma activators stimulate aquaporin 3 expression in keratinocytes/epidermis. Exp Dermatol 20:595-599.

Kaur S, Zilmer K, Leping V, and Zilmer M (2013) Serum methylglyoxal level and its association with oxidative stress and disease severity in patients with psoriasis. Arch Dermatol Res 305:489-494.

Kharaeva Z, Gostova E, De Luca C, Raskovic D, and Korkina L (2009) Clinical and biochemical effects of coenzyme Q(10), vitamin E, and selenium supplementation to psoriasis patients. Nutrition 25:295-302.

Kim NH and Lee AY (2010) Reduced aquaporin3 expression and survival of keratinocytes in the depigmented epidermis of vitiligo. J Invest Dermatol 130:2231-2239. Lee Y, Shin JM, Jang S, Choi DK, Seo MS, Kim HR, Sohn KC, Im M, Seo YJ, Lee JH, et al. (2014) Role of nuclear factor E2-related factor 2 (Nrf2) in epidermal differentiation. Arch Dermatol Res 306:677-682.

Moll R, Divo M, and Langbein L (2008) The human keratins: biology and pathology. Histochem Cell Biol 129:705-733. 
Mommers JM, van Rossum MM, van Erp PE, and van De Kerkhof PC (2000) Changes in keratin 6 and keratin 10 (co-)expression in lesional and symptomless skin of spreading psoriasis. Dermatology 201:15-20.

Peluso I, Cavaliere A, and Palmery M (2016) Plasma total antioxidant capacity and peroxidation biomarkers in psoriasis. J Biomed Sci 23:52.

Promsote W, Makala L, Li B, Smith SB, Singh N, Ganapathy V, Pace BS, and Martin PM (2014) Monomethylfumarate induces $\gamma$-globin expression and fetal hemoglobin production in cultured human retinal pigment epithelial (RPE) and erythroid cells, and in intact retina. Invest Ophthalmol Vis Sci 55: 5382-5393.

Qin H, Zheng X, Zhong X, Shetty AK, Elias PM, and Bollag WB (2011) Aquaporin-3 in keratinocytes and skin: its role and interaction with phospholipase D2. Arch Biochem Biophys 508:138-143.

Ramot Y, Sugawara K, Zákány N, Tóth BI, Bíró T, and Paus R (2013) A novel control of human keratin expression: cannabinoid receptor 1-mediated signaling downregulates the expression of keratins $\mathrm{K} 6$ and $\mathrm{K} 16$ in human keratinocytes in vitro and in situ. PeerJ 1:e40.

Stratis A, Pasparakis M, Rupec RA, Markur D, Hartmann K, Scharffetter-Kochanek K, Peters T, van Rooijen N, Krieg T, and Haase I (2006) Pathogenic role for skin macrophages in a mouse model of keratinocyte-induced psoriasis-like skin inflammation. $J$ Clin Invest 116:2094-2104.

Verkman AS (2008) A cautionary note on cosmetics containing ingredients that increase aquaporin-3 expression. Exp Dermatol 17:871-872.
Ward NL, Loyd CM, Wolfram JA, Diaconu D, Michaels CM, and McCormick TS (2011) Depletion of antigen-presenting cells by clodronate liposomes reverses the psoriatic skin phenotype in KC-Tie2 mice. Br J Dermatol 164:750-758.

Xie D, Seremwe M, Edwards JG, Podolsky R, and Bollag WB (2014) Distinct effects of different phosphatidylglycerol species on mouse keratinocyte proliferation. PLoS One 9:e107119.

Zenz R, Eferl R, Kenner L, Florin L, Hummerich L, Mehic D, Scheuch H, Angel P, Tschachler E, and Wagner EF (2005) Psoriasis-like skin disease and arthritis caused by inducible epidermal deletion of Jun proteins. Nature 437:369-375.

Zhang S, Liu X, Mei L, Wang H, and Fang F (2016) Epigallocatechin-3-gallate (EGCG) inhibits imiquimod-induced psoriasis-like inflammation of BALB/c mice. BMC Complement Altern Med 16:334.

Zheng X and Bollinger Bollag W (2003) Aquaporin 3 colocates with phospholipase $\mathrm{D}_{2}$ in caveolin-rich membrane microdomains and is downregulated upon keratinocyte differentiation. J Invest Dermatol 121:1487-1495.

Zheng X, Ray S, and Bollag WB (2003) Modulation of phospholipase D-mediated phosphatidylglycerol formation by differentiating agents in primary mouse epidermal keratinocytes. Biochim Biophys Acta 1643:25-36.

Address correspondence to: Wendy B. Bollag, Augusta University, 1120 15th Street, Augusta, GA 30912. E-mail: wbollag@augusta.edu 